AperTO - Archivio Istituzionale Open Access dell'Università di Torino

Exploring cultural heritage repositories with creative intelligence. The Labyrinth 3D system

This is a pre print version of the following article:

Original Citation:

Availability:

This version is available http://hdl.handle.net/2318/1578514

since 2017-12-04T09:32:41Z

Published version:

DOI:10.1016/j.entcom.2016.05.002

Terms of use:

Open Access

Anyone can freely access the full text of works made available as "Open Access". Works made available under a Creative Commons license can be used according to the terms and conditions of said license. Use of all other works requires consent of the right holder (author or publisher) if not exempted from copyright protection by the applicable law. 


\title{
Exploring cultural heritage repositories with creative intelligence. The Labyrinth 3D system.
}

\author{
Rossana Damiano ${ }^{1,1}$, Vincenzo Lombardo ${ }^{1,1}$, Antonio Lieto ${ }^{1}$ \\ ${ }^{a}$ Dipartimento di Informatica, Università di Torino \\ ${ }^{b}$ CIRMA, Università di Torino
}

\begin{abstract}
In cultural heritage, the use of ontologies makes the description of artworks clearer and self-explanatory, with advantages in terms of interoperability. The current shift towards semantic encoding opens the way to the creation of interfaces that allow the users to build personal paths in heritage collections by exploiting the relations over the artworks.

In the attempt to leverage this multiplicity of paths, we designed and implemented a system, called Labyrinth 3D, which integrates the semantic annotation of cultural objects with the interaction style of 3D games. The system immerses the user into a virtual 3D labyrinth, where turning points and paths represent the semantic relations over cultural objects, with the goal of engaging the user in the exploration of the collection.
\end{abstract}

Keywords: 3D visualization, cultural heritage, computational ontologies

\section{Introduction}

In the last decade, the advent of connected, portable devices, and the evolution of the Web towards a participatory model have prompted cultural institutions to pursue new communication strategies that leverage the Web $[1,2]$. Cultural institutions have rushed to publish their collections online, with the goal of innovating their interaction with the audience through the help of personalization and social media $[3,4]$.

In parallel with this trend, digital archives have moved towards semantic annotation, a paradigm where the items in the archive are described with reference to a computational ontology. The use of ontologies, implemented through logic-based languages [5], makes the description of artworks clearer and unambiguous, with advantages in terms of interoperability among systems $[6,7]$. Semantically annotated collections, then, lend themselves to personalization [4] and cross media integration of data sources, following the paradigm of Linked Open Data $[8,9]$.

Despite the potential of the semantic representation, however, the search in heritage archives is still largely based on keywords and/or tags, through which users can filter the archive contents to find what they need. As exemplified 
by the well known Europeana initiative, which provides a unified interface to a set of national digital collections [10], the search typically returns a list of items (books, pictures, videos, etc.) accompanied with personalized recommendations, but it does not contain an explicit representation of meaning relations over them. In contrast with this approach, [11] argues that, in order to meet the needs of the general audience, tools for supporting exploratory search are needed besides the traditional keyword-based interfaces. In cultural heritage, search interfaces are typically based on the metaphor of the "archive", which mirrors the actual fruition of the physical cultural objects (see, for example, the web interface of the above mentioned Europeana system), although the trend of the 3D "visit" has emerged in online museum collections, as demonstrated by the well known Google Art Project. ${ }^{1}$

In this paper, we address the access to digital collections by proposing an approach that leverages semantic annotation to create a 3D environment where the user can explore the semantic relations over the items in a visual environment. Our approach combines the use of the 3D language, typical of new media - and video games in particular -, with the capability of semantic annotation to connect entities that are distant in space and time but share some common features at the cultural level. The use of $3 \mathrm{D}$ for the interface is motivated by the goal of attaining a user experience characterized by high level of engagement and a sense of immersion [12]. As shown by an established line of research in information visualization [13], in fact, visual metaphors can convey a conceptual model in an immediate and engaging way.

The system we describe in this paper is part of a larger project, called Labyrinth $^{2}$, aimed at the dissemination of cultural heritage archives to the general audience. In order to mediate between the point of view of the user and the heterogeneity of the items in heritage repositories, which usually differ by features such as media type, age and purpose but share some narrative features like stories and characters, the project relies on the notion of "archetypes" of narrative nature. Mainly inspired by the research in iconology and narratology $[14,15]$, the term "archetype" is employed in Labyrinth to refer to a conceptual core set at the intersection of narrative motifs, iconological themes and classical mythology (the system itself is named after a well-known archetype).

The plan of the paper is the following: after describing the background of the project and discussing its motivations (Section 2), in Section 3 we provide a brief overview of the live system with a navigation example. Section 4 describes the components of the systems, namely the ontology (Section 4.1), the 3D environment (Section 4.2) and the core component of the system, i.e., the mapping of the ontology onto the 3D environment (Section 4.3). The system architecture, which combines these elements to create newer and newer paths through the repository, is described in Section 5. Discussion and conclusion end the paper.

\footnotetext{
${ }^{1}$ https://www.google.com/culturalinstitute/u/0/project/art-project

${ }^{2}$ http://app.labyrinth-project.it:8080/LabyrinthTest/
} 


\section{Background}

In the last decade, the use of ontologies for the access to cultural heritage collections has been investigated by several projects. A pioneering contribution was given by the Finnish Culture Sampo project [16]. In this project, a number of domain ontologies provide the background against which cultural objects (including artworks, artists, traditional practices, etc.), encoded in different media formats (e.g., images and videos), can be explored, tracking the underlying relations over them. In CultureSampo, once a certain artifact (e.g., a painting) has been retrieved, it is possible to explore the relations over the objects (and characters) represented therein. The system has recently evolved towards a linked data approach with the release of a new application, War Sampo, focused on the Second World War [9]. The Agora system [17] frames the exploration of a digital collection into historically relevant episodes, supported by a semantic account of the notion of event [18]. For example, the user can choose a historical episode (e.g., "German occupation of Poland in the Second World War") and navigate among the cultural objects related to this event.

A line of research in ontology-based systems has specifically explored the use of narrative models in cultural heritage dissemination. Stories not only represent an effective way to convey information in a compact format, as argued by [19], but, according to the research in cognitive psychology, they are a primary means for the conceptualization of reality [20]. In cultural heritage, many artworks have, by and large, some type of narrative content. In visual arts, for example, paintings often display story episodes while statues immortalize characters; even non representational artworks often refer to narrative elements, despite the abstract nature of their visual content. Stories are narrated by textual media such as tales and novels, but also - though in nonverbal terms - by different kinds of musical works, from operas to symphonic poems. Narrative is the focus of the Bletchley Park Text system [21], a semantic system designed with the goal of supporting the users in the exploration of online museum collections. Designed with the notion of the "guided visit" in mind, the system encompasses an ontology of story, taken from the Story Fountain project [22]. The stories represented in the system are employed in a web interface to create relations over entities in online collections; based on this knowledge, the user can ask the system to find a narrative connection between different entities. More recently, the Decypher EU project leverages stories to addresses the curatorial side of cultural heritage dissemination [23]. In Decipher, a story ontology is the basis of a system that supports the creation of story-based collections by museum curators. Finally, Europeana also uses some simple narrative features to describe the items they contain. In Europeana, it is possible, for example, to navigate among the artifacts representing a given action or displaying a certain character, across a large number of indexed objects; the system does not provide, however, a story-level navigation.

The Labyrinth project extends the approaches described above by integrating the use of a narrative model to connect the items in a collection with the use of a visual environment for the exploration of these connections. The system re- 
lies on an ontology of narrative archetypes to describe the items in the collection; the $3 \mathrm{D}$ interface of system is inspired to a well known narrative archetype, the "labyrinth". The notion of labyrinth is not only deeply rooted in the Western Culture, dating back to Greek Myths and witnessed by several archaeological locations across Europe [24], but also, thanks to the graph like nature of the notion of labyrinth [25], it lends itself well to representing the many-to-many relations among artworks encoded in the ontology. The goal of the visual design of the 3D interface is two-fold: on the one side, it is aimed at engaging the users to explore the repository though an immersive experience; on the other side, it is aimed at making the system usable by the large majority of uses by integrating information giving and entertainment in a familiar environment. The labyrinth, or maze, is a genre of video games most users are familiar with, thanks to classic 2D games such as Atari's Pacman ${ }^{3}$ and recent 3D titles such as Imangi's Temple Run $^{4}$ or PlayFirst's Dream Chronicles ${ }^{5}$.

In cultural heritage, the use of $3 \mathrm{D}$ visualization is normally intended as a support for study and dissemination activities. 3D projects in cultural heritage can be roughly divided into two types: virtual equivalents of physically existing locations, such as museums and historical buildings, and reconstructions of physical environments that have disappeared, such as archaeological locations or temporary art works. Google Art Project ${ }^{6}$ and Arounder ${ }^{7}$ are examples of the first type, where 3D is often obtained through PMVR techniques that integrate high definition images of artworks in the 3D environments. Rome Reborn [26], the $3 \mathrm{D}$ reconstruction of Rome as it appeared in the IV century, is an example of the second type. In this project, the use of 3D is integrated with animated characters of ancient Romans, who interact with the users. A similar approach is proposed by [27], who present a framework for 3D real time applications in web browsers, employed to develop virtual reconstructions of Rome (Virtual Rome project) and other Italian locations [28]. Labyrinth differentiates from these approaches since the 3D representation is not employed to reconstruct real environments or to create virtual ones, but as a tool to convey semantic relations through a visual environment. For this reason, the system does not encompass a semantic model of the 3D environment: rather, it maps a semantic representation of the domain onto the 3D environment, as part of the interaction design process.

\section{Live System}

The Labyrinth project encompasses both a standard web based interface [29] and a 3D application [30]. Both interfaces allow the user to navigate a repository of cultural objects with the guidance of a set of archetypes of narrative nature.

\footnotetext{
${ }^{3}$ https://en.wikipedia.org/wiki/Pac-Man

${ }^{4}$ http://www.imangistudios.com

${ }^{5}$ http://www.playfirst.com/games/view/dream-chronicles

${ }^{6}$ www.google.com/culturalinstitute/project/art-project

${ }^{7}$ www.arounder.com
} 


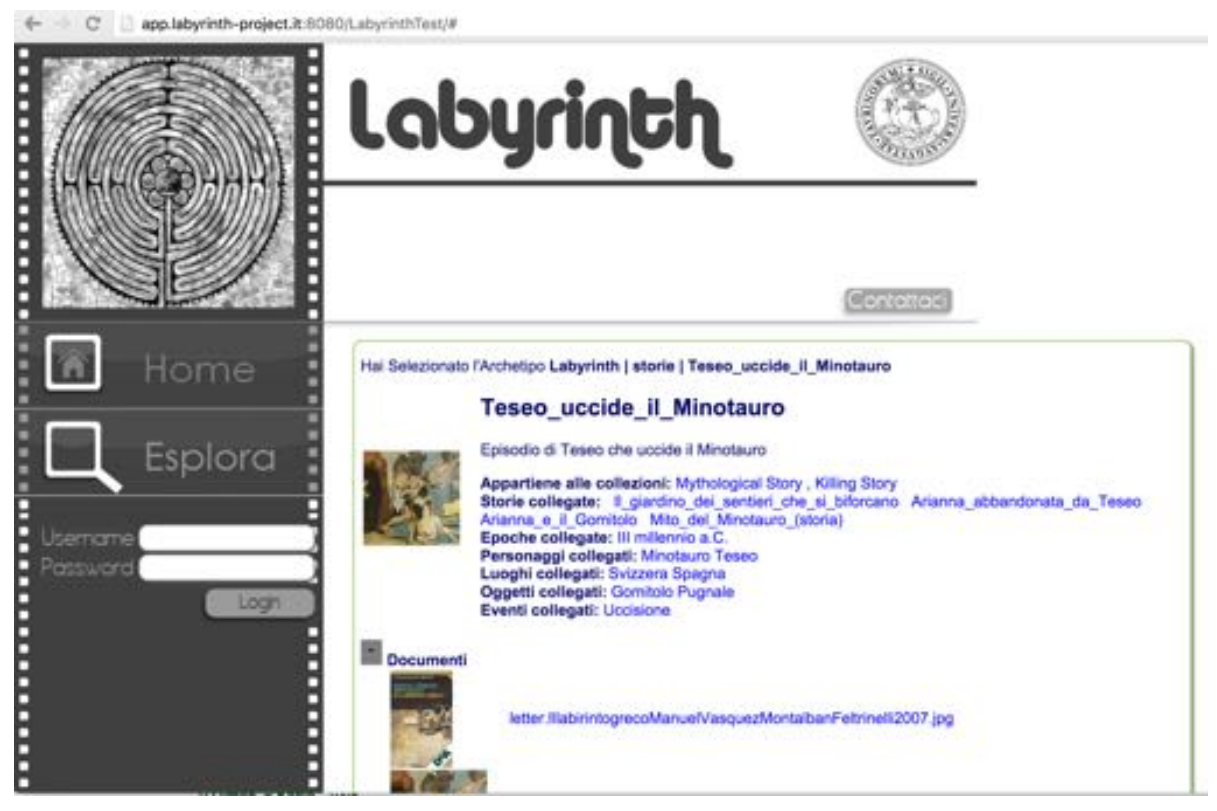

Figure 1: A screenshot of the web interface of the system (in Italian).

The archetypes are contained in an ontology that describes each archetype in terms of its related stories, characters, objects, events, and locations, and stores the connections that relate these categories with the items in the repository.

In both the hypertextual and the 3D interfaces, the interaction with the user starts with the selection of an archetype. In the hypertextual interface, the users continues by refining her/his search based on the inner articulation of the selected archetype into more specific categories (namely, stories, characters, objects, events, locations and epochs), then into single elements within the category (single story, character, etc.), by following a top-down strategy that ends with the selection of a specific artifact (for a detailed description, see [31]). Fig. 1 shows a screenshot of the interface (in Italian): after selecting the archetype of the labyrinth, the user has decided to explore the category of "stories", then the specific story entitled "Theseus kills the Minotaur". As a result, the interface shows a record of the story (upper part of the main box, "Teseo uccide il Minotauro"), which includes the related stories, the characters and objects featured by the story, and the locations and epochs in which the story takes place. The user can click on them to navigate from the currently selected story to another, or to move to a different category of the archetype (for example, "characters" or "locations"). Below (bottom of Figure 1), the interface shows the thumbnails of the artifacts (or, better, of their digital copy) that refer to the currently selected element (here, the story "Theseus kills the Minotaur"); each thumbnail can be clicked on to get a record of the artifact. 


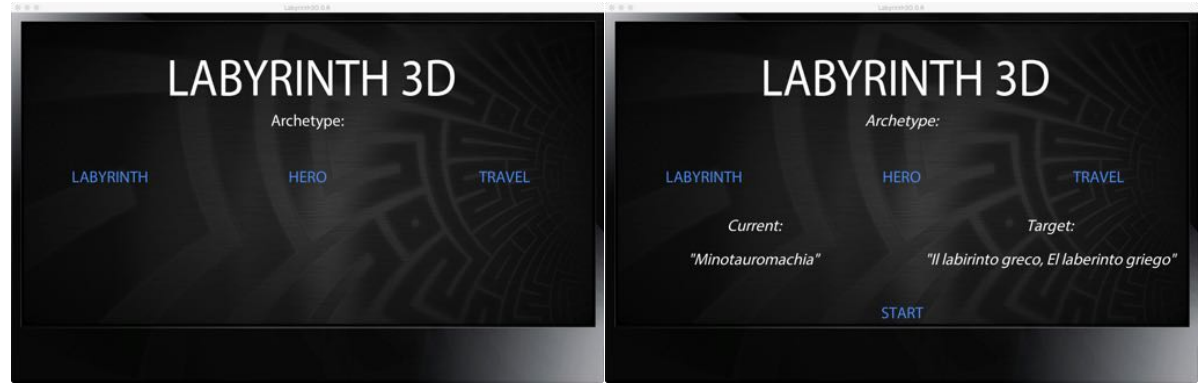

Figure 2: 3D interface: selection of the archetype (left) and assignment of initial and target artworks (right, labeled as "current" and "target").
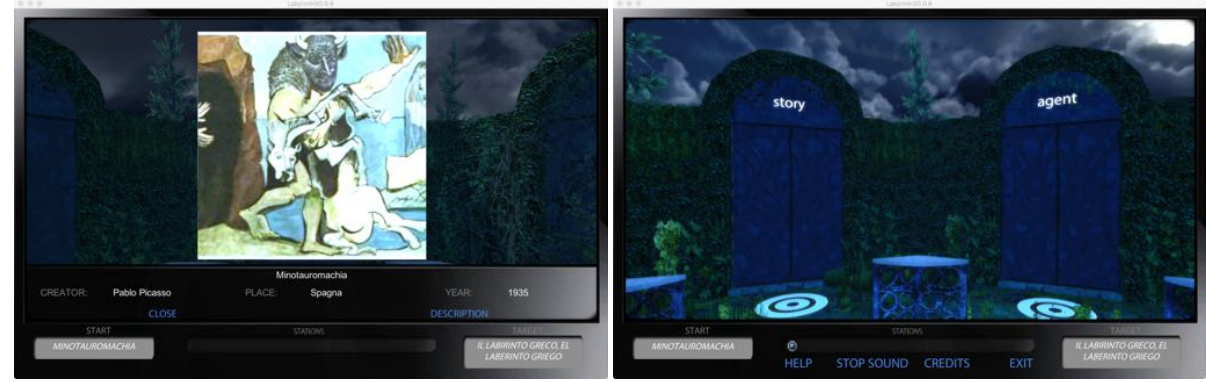

Figure 3: First step of the navigation: the initial artwork, Minotauromachia (left); right: some of the doors available from the initial artwork (same character and same story).

A slide show of the thumbnails is positioned on the left of the story record to provide a quick glance on the available contents for the current selection.

Differently from the hypertextual interface, the 3D interface is characterized by a bottom-up approach: here, the user navigates from artifact to artifact on the basis of the relations over them represented in the ontology, building her/his own personal path through the repository. The user is situated in a virtual maze where the artworks are located in the clearings and connected by pathways that represent the relations over the artworks. Immersed in the virtual maze in a first person perspective, the user is encouraged to explore the repository in the same way as the visitor of a hedge maze explores the turns and twists of the maze on her/his way to the exit.

In order to exemplify the user experience in the 3D labyrinth, we will describe a navigation example extracted from the system log, illustrated through the screenshots of the steps that compose it (Figures 4, 5, 6). After choosing the archetype of the "labyrinth" (Fig. 2, left), the user is assigned a start and a target artwork (Fig. 2, right), randomly extracted from the repository: in the example, they are, respectively, the "Minotauromachia" (a painting) and a novel, "Il labirinto greco". When the user clicks on the Start button posited below the start and target nodes, she/he is brought to the $3 \mathrm{D}$ environment 

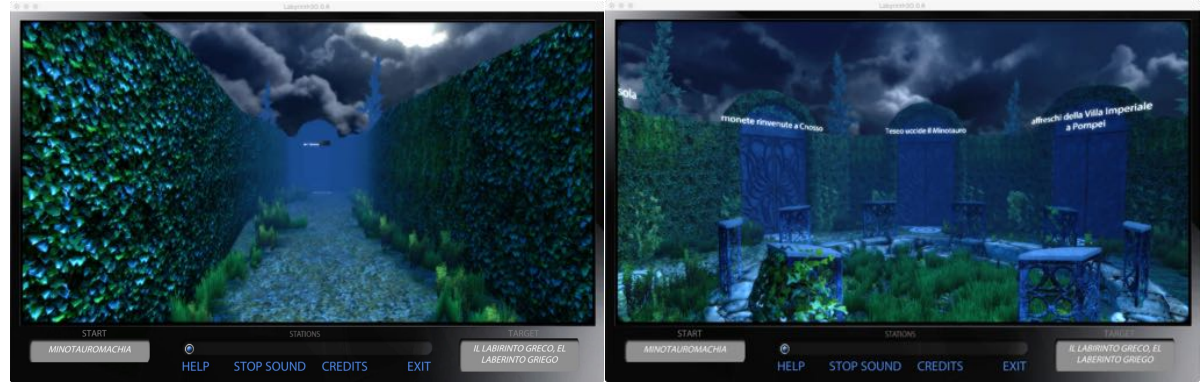

Figure 4: Second step of the navigation: a pathway (left) to the subsequent artwork; second artwork (right), with doors leading to the other artworks in the "same character" relation.

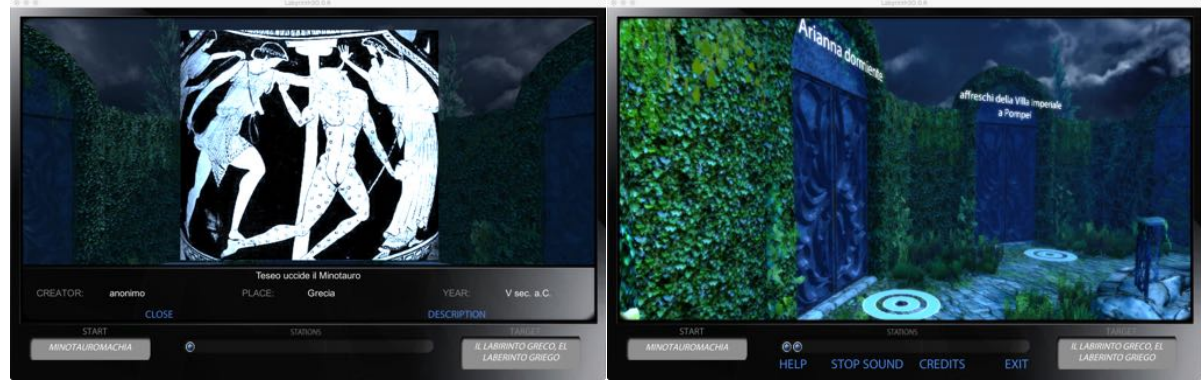

Figure 5: Third step of the navigation: a Greek vase representing Thesues killing the Minotaur (left); doors leading to the artworks referring to the same story (right).

(Fig. 3, left). The first location is the node containing the start artwork, the painting entitled "Minotauromachia" by Pablo Picasso, which shows the Greek hero Theseus fighting with a Minotaur. Fig. 3 (left) shows how the artwork (here, a picture of the painting) is displayed to the user in a 2D layer temporarily superimposed to the 3D scene; the artwork is accompanied by the information about its author, the place where it is hosted and the creation date. A longer description can be obtained by clicking on "description", below the image; by clicking on "close", the layer disappears. Figure 3 (right) shows some of the connections available from the node, represented by the two doors labeled as "agent" and "story" (other doors are out of the view): the first door leads to artworks that feature the same character (named "agent" in the system) as the current artwork, the second door leads to artworks that relate to the same story.

By choosing the door labeled as "agent", the user is led through a pathway (Fig. 4, left) to an empty node (Fig. 4, right) that contains doors for the artworks that display the same character as the previous artwork, Theseus; the titles of the artworks are written above the doors, from left to right: "Monete rivenute a Cnosso" ("Coins found in Knossos"), "Teseo uccide il Minotauro" ("Theseus kills the Minotaur"), "Affreschi della villa imperiale a Pompei" ("Frescos, Villa Imperiale in Pompei"). The user selects the middle door, and 


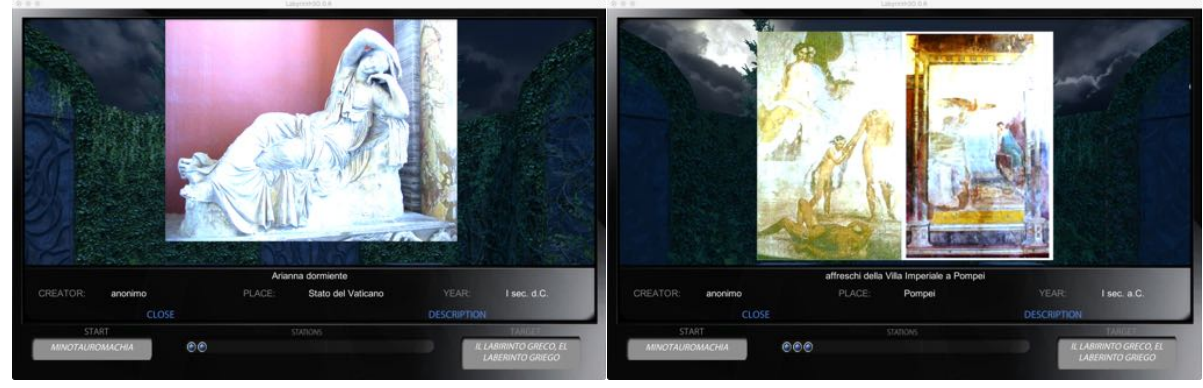

Figure 6: Left: fourth step of the navigation, a statue of Ariadne (same story relation); a different artwork (right), reachable by backtracking to the previous step (a Roman fresco representing the myth of the Minotaur in Pompei).

is led to a node that contains a Greek vase displaying the Theseus in the act of killing the Minotaur (Fig. 5, left). Notice that the console posited in the bottom part of the interface contains, besides the controls for getting help, stopping the sound and exiting the application, a progress bar displaying the artworks visited by the user so far: by selecting a previously visited artwork, the user is brought back to the node containing it. After the Greek vase, the user follows the same story relation by clicking on the door labeled as "story" (not shown), and is brought to an empty node with doors for the artworks that refer to the same story: "Arianna dormiente" ("Sleeping Ariadne") and "Affreschi della villa imperiale a Pompei" ("Frescos, Villa Imperiale in Pompei": notice that, like in a true labyrinth, the same artwork can be gained by following different paths). By choosing the first door, "Arianna dormiente" ("Sleeping Ariadne"), the user will reach a node containing a Roman statue of Ariadne, the female character of the myth of the Minotaur (Fig. 6, left); from there, by backtracking to the previous node, the user may select the second door, "Affreschi della villa imperiale a Pompei" ("Frescos, Villa Imperiale in Pompei"), which leads to a node containing a painting that illustrates the myth of the Minotaur, located in a Roman villa in archaeological site of Pompei (Fig. 6, right).

\section{The tripartite core of Labyrinth}

Given a collection of cultural objects, commonly represented by the digital resources that constitute the "digital equivalents" of the actual physical objects [32], the access to the collection in an ontology based system such as Labyrinth $3 \mathrm{D}$ is the result of the interplay of three elements, namely the information about the objects, or metadata, contained in the ontology (Section 4.1), by which the objects are indexed, the visualization interface (Section 4.2), driven by the project specific goals (dissemination, presentation, study, etc.), and the mapping of the objects onto the visualization interface (Section 4.3). Thanks to this tripartite relation, the system translates the information about the cultural objects into a visual representation where the semantic relations over the objects contained in the ontology are mapped onto the elements of a $3 \mathrm{D}$ environment. 


\subsection{The Archetype Ontology}

The description of the artworks encoded in the metadata typically includes features such as date, authorship and title of the items, normally expressed according to standard vocabularies, such as ISO 8601 for dates ${ }^{8}$ or ULAN (Union List of Artists Names) for names. ${ }^{9}$ Beside authorship and editorial information, metadata usually contain also information about the management and preservation of cultural objects, such as responsibility for the preservation, digitalization standards, etc. In the last decade, metadata have evolved towards semantic encodings describing the content of the artworks, with categories such as iconography, event types, etc.[33, 34]. An example of semantic description is provided by the Europeana Semantic Model (ESM) ${ }^{10}$.

As exemplified by the navigation example provided in the previous section, the semantic annotation of the artworks in Labyrinth is mainly oriented to the representation of their content, and narrative content in particular. The narrative content of the artworks is expressed through a set of archetypes that characterize Western culture through the ages, heritage of the Greek and Roman tradition [35]. The core of the Labyrinth system is the Archetype Ontology (AO), described in detail in [31]. The AO contains a number of archetypes (the journey, the labirinth, the hero) and describes how the artworks relate to them via the representation of stories, characters, objects, events, locations and epochs. The AO contains 8 top classes: the Archetype class contains the archetypes; the Artifact class contains the artworks, organized according to the FRBR model [36]; Entity contains the characters and objects represented in artifacts; Story represents a collection of stories related with the archetypes; the Description Templates class contains a role-based schema for describing events and states that can be filled by characters and objects; the Format class encodes the format and type of media resources; Geographical Place and Temporal Collocation, finally, encode, respectively, the spatial and temporal information related to artifacts, stories and archetypes. The Archetype Ontology was manually built based on an extensive survey of the notion of archetype, spanning from Warburg's Bilder Atlas [14] to folkloric studies [15] and contemporary accounts of tropes in media. ${ }^{11}$ The ontology was aligned with the conceptual reference model established by the International Council of Museums (ICOM), the CRM-FRBR model [36], a standard in the description of cultural heritage.

In the Labyrinth system, the editing phase is conducted through a back-end web interface through which items can be added to the repository. The description of the items is accomplished through form filling and it follows the Dublin Core initiative [37], a metadata element schema that has become a standard de

\footnotetext{
${ }^{8}$ http://www.iso.org/iso/home/standards/iso8601.htm

${ }^{9}$ http://www.getty.edu/research/tools/vocabularies/ulan/

${ }^{10}$ http://pro.europeana.eu/ese-documentation

${ }^{11}$ http://tvtropes.org
} 
facto in digital archives ${ }^{12}$. When a new item is added to the repository, the system imports the description of the item in the ontology through a built-in procedure that converts the input data into the ontology format (a set of $\mathrm{RDF}^{13}$ triples). First, the internalization phase (described in details in [29]) translates the metadata of the resource (creator, date, etc. ) into the language of the ontology. Then, a mapping procedure matches the imported description with the available archetypes. Both steps are achieved via if-then rules encoded in SWRL ${ }^{14}$, the rule language designed for ontologies. As an example of how the mapping is accomplished, consider the rule that examines the "title" metadata element of an artwork in order to find a connection with the archetype of the "Labyrinth": if words like "labyrinth" or "maze" are found in the title, the rule will add to the ontology the assertion that the artwork evokes the archetype of the labyrinth. Finally, after the new item has been internalized in the system and mapped onto the ontology, a specific set of rules add the narrative features to the artworks (narrative mapping). For example, if an artwork represents a set of characters performing some action, the system searches for a story in which the same characters perform that action (see [29] for a detailed description of how the narrative properties are added to the representation of the items). For instance, an artwork representing Ariadne in the act of giving the ball of thread to Theseus (a focal event in the myth of the Minotaur) would be recognized as having the myth of the Minotaur as a narrative component.

In order to illustrate how the items in the repository are represented in the ontology as a result of the internalization process, we will resort to an example. Fig. 7 illustrates the description of the painting "Minotauromachia" by Pablo Picasso (the first step of the navigation example in Section 4.1), serialized in the XML/RDF format. Notice that each line represents an RDF triple, composed of a subject (all triples in this fragment have the same subject, i.e., the named individual "Minotauromachia" in line 2), a predicate (for example, hasResource Type, line 4) describing a property of the subject or a relation with another entity, and an object (here, the resource type, Image) which constitutes the value of the property - or the second term of the relation. All resources are characterized by a prefix given by the URI of the ontology. Fig. 7 outlines the role of each phase of the procedure described above. The annotation is divided into two groups of assertions: the first group contains the assertions extracted from the artwork metadata by the internalization procedure; the second group contains the properties added by the mapping procedure, which connects the artwork with the archetypes: this group contains specific annotations concerning the narrative relations among the artworks.

Lines 3 to 6 are created by the internalization procedure and correspond to the artwork metadata, such as type, creator, etc. The hasResourceType property (line 4) describes the media type of the resource, i.e., image; the hasCreator

\footnotetext{
12https://www.iso.org/obp/ui/\#iso:std:iso:15836:ed-2:v1:en

13 https://www.w3.org/TR/rdf-concepts/

${ }^{14}$ https://www.w3.org/Submission/SWRL/
} 
Properties added by the internalization phase:

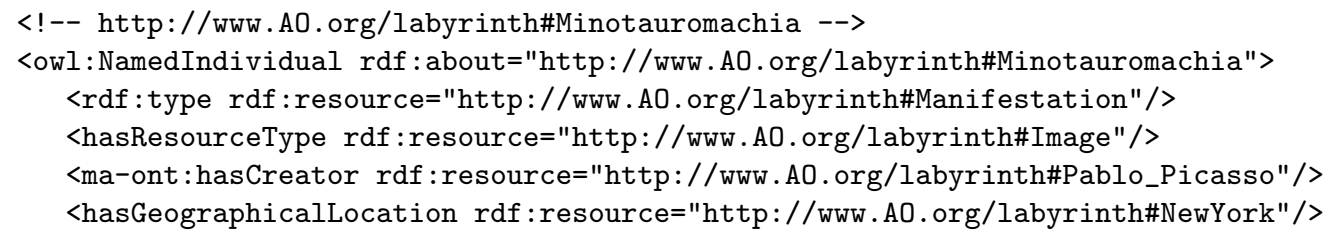

After the mapping phase:

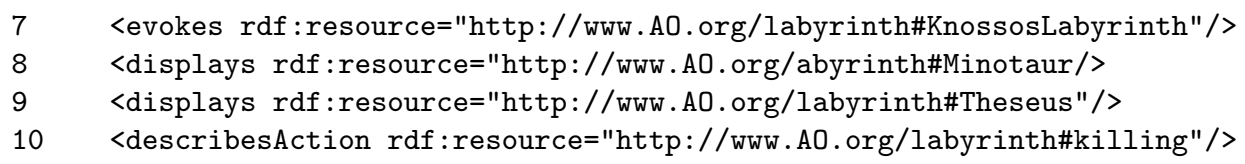

After the narrative reasoning:

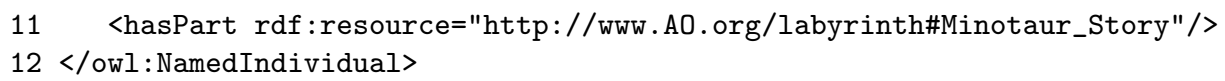

Figure 7: The description of the artwork "Minotauromachia" by Pablo Picasso in the AO ontology. The sections show the properties added by each phase of the internalization and mapping procedures.

property (line 5) connects the painting with its author, "Pablo Picasso"; the hasGeographicalLocation property (line 6) describes the location of the artwork. Lines 7 to 11 describe the relation of the artwork with the archetype: the property evokes (line 7) relates the painting with the archetype of the "Labyrinth", while a set of specific properties describe the relation with the archetype in greater detail, focusing on its narrative aspects: displays (lines 8-9) refers to the characters which appear in it, i.e., Theseus and the Minotaur; describesAction (line 10) refers to the event type it depicts ("killing"). Finally, the property hasPart (line 11) states that the painting contains, as part of its narrative content, the Minotaur Story.

Given this description, several relations can be detected with other artworks. Besides the standard relations based on author or resource type, the archetype of the labyrinth connects the artwork with other artworks that display the same characters (Theseus or the Minotaur), depict the same action type (killing), or refer to the same story (the myth of the Minotaur) and other related stories (e.g., Ariadne and the Thread).

\subsection{Designing the $3 D$ environment}

The design of the 3D environment is inspired by the metaphor of the labyrinth. This metaphor was chosen for its ability to convey the graph-like nature of the relations over the artworks in a cultural heritage collection, and for its immediacy of use, since it provides an intuitive mapping for artworks (nodes of the 
labyrinth) and relations over them (connections among the nodes). The interaction metaphor underlying the navigation is "finding one's way": here, however, the user does not simply gain the exit, but the creation of a personal path in artworks' meaning, represented by a virtual "red thread". In order to make the experience more engaging, when the session begins, the user is given a target node. When the user reaches the target node, or when the user decides to exit from the labyrinth, the session ends and the user is shown the statistics about her/his own path: number of visited nodes, elapsed time, backtrackings, etc.

The visual design of the labyrinth is inspired to the classical hedge maze, with architectural elements that are intended to remind of some distant but indefinite past; this choice was primarily due to the constraint posed by the heterogeneity of the contents assumed by the project. The floor is partly tiled, partly covered with grass, and the mood is inspired by a dark, Gothic style. The maze contains two types of nodes: some nodes (artwork nodes) contain artworks, some nodes (relation nodes) are empty and only serve the function of connecting the artwork nodes, as exemplified in the navigation example provided in Section 3 . The presence of an artwork in a node is signaled by a low circular balustrade in the middle, open in several points, that are intended as affordances inviting the user to step into the inner part of the node [38]. The entrance to pathways is marked by doors; each door corresponds to a semantic connection (e.g., same story), and is surmounted by the name of the type of connection (e.g., "story"). Each node has a fixed number of doors/pathways: depending of the number of semantic relations that connect the node with other nodes, some doors may be closed, or hidden by greenery. If the connection leads straight to a single artwork, the title of the artwork is posited above the door. Pathways differ in length and form: some are short, some are longer and they bend, so that their end is not visible, in order add some thrill to the experience.

The navigation in the system is inspired to the paradigm of constrained navigation [39], with the aim of making it usable also for non expert users of 3D applications. The user moves by clicking on small circles of light posited on the floor, in front of the doors of the nodes and along the pathways. Circles of the same type also mark the presence of an artwork in the middle of artwork nodes and must be clicked to get information about the artwork. Smaller circles of light appear inside the circles when they are clicked, so that they eventually form a sort of "red thread" that marks the path made by the user so far. The metaphor of the red thread, aimed at improving self orientation, is enforced also by the console posited in the lower part of the screen, that shows the list the nodes visited by the user. By clicking on a node in the list, the user is brought back to that node. The console also contains buttons for ending the session and turning off the sound. The user is free to explore the labyrinth, going back to previous locations and clicking on the control posited in artwork nodes to receive information and experience them via the appropriate plugins: depending of the media type of the resource associated with the artwork, an image is displayed, a video is played, etc. A short description of the artwork, with title, date and creator, is always provided, as exemplified in Fig. 3 (Sect. 3). 
The 3D environment was implemented with the Unity $3 \mathrm{D}^{15}$ real time engine, which supports several platforms and mobile conditions. Unity $3 \mathrm{D}$ offers first person gameplay default assets, both concerning camera motion control and mouse tracking motion control. In order to optimize the production time and cost of the 3D assets, a single model of the labyrinth node, with a predefined set of exits, was created: at run time, it is dynamically adapted to the semantic relations connecting the current artwork with the others by closing or opening the corresponding number of doors. To achieve our goals, we built an indexed database of 3D objects to be promptly displayed in real time by the 3D Engine. Thus, we were able to produce several theme variations exponentially exploding the number of possible combinations. The standard 3D objects are: the octagonal square (3 variants, actually), the open door (3 variants), the closed door (2 variants), the textual artwork viewer (1 variant), the pictorial artwork viewer (1 variant), the movie viewer (1 variant). The pathways are a $3 \mathrm{D}$ object category on their own: they vary in shape in accordance to their length, which is measured in steps (2-3-4 steps, each in three variants). Joined together, steps compose asymmetrical paths, which can also be used backwards, therefore multiplying the possible combinations of subsequent pathways. So, the maze, determined through the user choices, is perceived as never being the same.

\subsection{Mapping the ontology onto the $3 D$}

The mapping of the semantic relations onto the visual environment poses some problems that need to be faced as part of the system design, and constrain the architecture of the system. Formally, the labyrinth is an undirected graph [25], where vertices have a variable degree. ${ }^{16}$ The nodes correspond to the graph vertices, the pathways to the edges. Notice that, as in a real maze, there are also nodes with only one incident edge, i.e. dead ends where the user has to backtrack. The direct transposition of the graph-like structure of the relationships over the artworks from the ontology to the 3D labyrinth, however, would lead to a proliferation of the edges that would be confusing for the user. Take, for example, the similarity relation "displaying the same character" among artworks. Representing this relation as artwork to artwork relations implies that, for each artwork, an edge should be added from the artwork to every other artwork that displays the same character (and this should be done for each semantic relation). In order to alleviate this problem, in Labyrinth, we decided to represent the semantic relations such as "displaying the same character" through special nodes that represent the relation itself, thus obtaining a more compact representation. These nodes do not correspond to artworks, but simply distribute the semantic relation over the pairs of artworks that are in the given relation.

\footnotetext{
${ }^{15}$ https://unity3d.com

${ }^{16}$ For usability reasons, the maximum number of edges per vertex has been limited to the arbitrary threshold of 7 , given the well known limitations of short term memory first shown by Miller [40].
} 


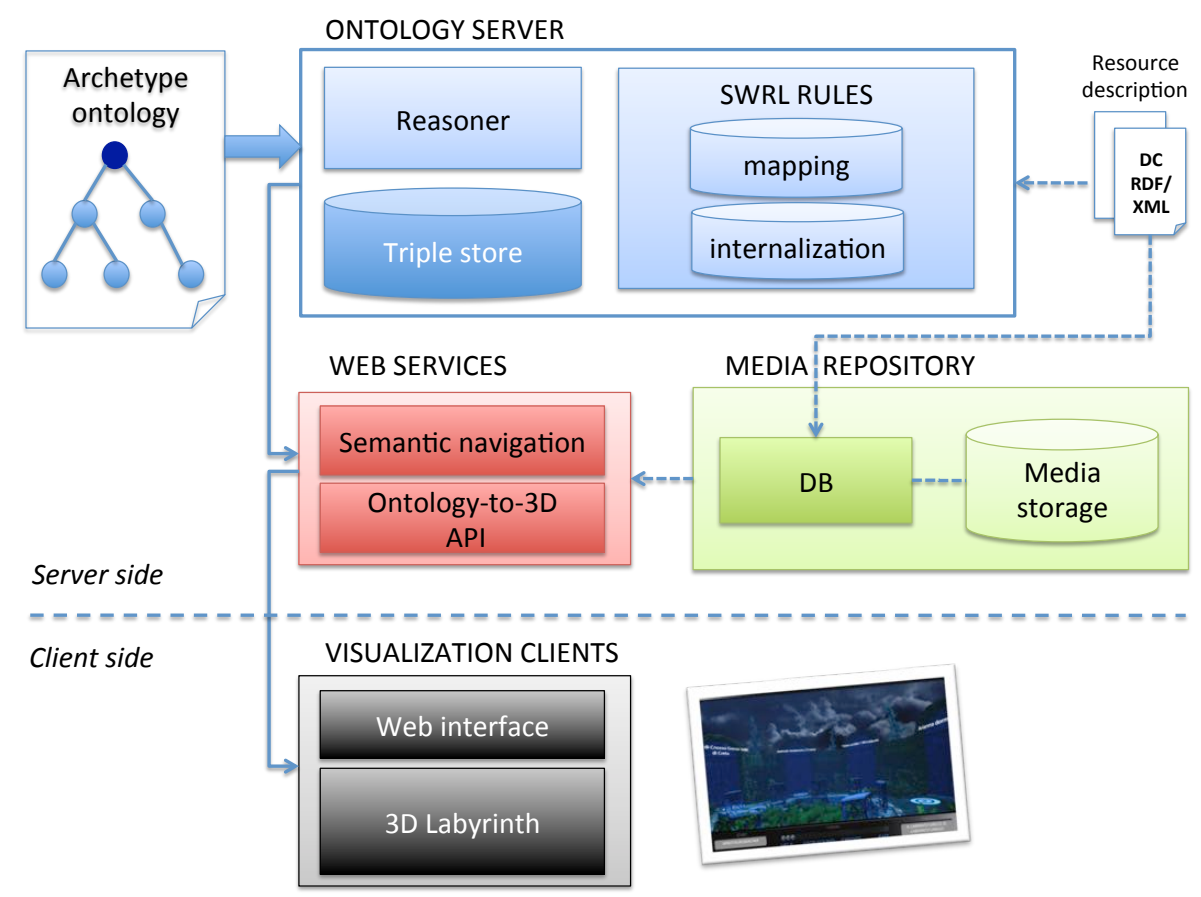

Figure 8: The architecture of the Labyrinth system.

As a result of the constraint described above, there are two types of nodes (with different iconic elements) in the labyrinth, connected by the pathways: artwork nodes and relation nodes. Artwork nodes are connected with both relation nodes and artwork nodes. Relation nodes are connected only with artwork nodes. The user navigation starts from an artwork node: the user has to choose one of the pathways exiting from the node, labeled either with the name of a different artwork (in this case, the pathway, leads directly to an artwork node) or with the name of a semantic relation (in this case, the pathway leads to a relation node, that in turn leads to a set of different artwork nodes). Since the semantic relations are symmetric, pathways can be walked both ways.

\section{The Labyrinth system}

In this section, we describe the architecture of the labyrinth system, which constructs the $3 \mathrm{D}$ environment as long as the interaction with the user progresses.

\subsection{System architecture}

The architecture of the Labyrinth system is structured according a clientserver schema. The ontology is stored in an ontology server; the information it 
contains is dynamically extracted from the ontology and made available to the visualization client, which manages the user interface.

The system encompasses four main modules (see Fig. 8):

- the Ontology Server (Fig. 8, top) stores the AO ontology - where the cultural heritage objects are described - and provides the reasoning services that allow the system to establish the relations of each object with the archetypes, as exemplified in Section 4.1 (for example, inferring the relation between an artifact and a story given the characters displayed in the artifact). In the current implementation, the ontology server is provided by Owlim. ${ }^{17}$ The ontology server also supports the SWRL rule sets that implement the internalization and mapping procedures described in Section 4.1, by which new items are ingested in the system. The ontology server provides also the SPARQL ${ }^{18}$ endpoint for querying the ontology, necessary to extract from the ontology the data that will be visualized in the interface (i.e., the semantic relations over the artifacts, such as the same character or the same story relations exemplified in the navigation example in Sect. 3). Notice that this module is independent of the visualization type and it serves both the web-based interface and the 3D environment.

- the Media Repository (Fig. 8, right) stores the media objects (the digital equivalents of the artworks) which constitute the repository of the system and is indexed by a relational database (a mySql database);

- a set of Web Services (Fig. 8, left) implement the Application Programming Interface specific to each visualization client. This component extracts the data from the ontology in response to the requests of the clients. The web services, written in Java, are called by the visualization clients to respond to the actions of the user, and return the data in XML format. For example, in the 3D environment, when the user clicks on a door leading to a given artwork, the visualization client calls the API command that fetches the information about the artwork, needed to generate the node with the artwork in the $3 \mathrm{D}$ maze.

- the Visualization Clients (Fig. 8, bottom) support the interaction with the user through 3D navigation, as standalone application (for the 3D system) or embedded in a web browser (for the web interface).

The core of the system consists of the APIs that fetch the data from the ontology to the visualization clients. The interplay among these components realizes the mapping of the objects and relations encoded in the ontology onto the environments where they can be visualized by the user. In the following, we describe in detail the interaction between the Ontology-to-3D API and the 3D

\footnotetext{
${ }^{17}$ http://www.ontotext.com/products/ontotext-graphdb-owlim-new-2/

${ }^{18}$ https://www.w3.org/TR/sparql11-overview/
} 
visualization client. The interaction between these two components achieves the computational creativity that the user can enjoy by navigating the repository in the virtual maze.

\subsection{The system at work}

The topology of the maze is computed locally as the user progresses in her/his path. This choice is partly related with the user experience design and partly related with optimization issues. Concerning the user experience, the step by step generation of the maze provides room for the adaptive personalization of the navigation experience, which can be tailored to the typology and behavior of the user given the available relations over the artworks. Currently, the variability of the navigation is provided by a basic random mechanism. Since the system does not pose any constraints on the number of related artworks, the available relations over them may exceed the number of doors, set to 6 in the current implementation, when necessary, 6 artworks are randomly extracted. As a result, in different navigation sessions, different artworks may be extracted, thus generating slight variations in the user experience. ${ }^{19}$ Concerning the optimization issues, the step by step generation of the topology guarantees that the computation needed to generate the maze is not affected by the size and the connectivity degree of the repository (which only affect the execution of the queries). ${ }^{20}$ Moreover, if the repository changes, no initialization is needed. Notice also that this solution is made possible by the fact that the $3 \mathrm{D}$ environment does not encompass a top down, map-like visualization of the maze, with the consequence that the user can only experience a subjective view of the environment. This choice, although debatable for the lack of orientation it may provoke in the user, is consistent with the actual experience of the real hedge maze by which the design of the 3D system is inspired.

Basically, the maze is generated on the fly as follows: when the navigation begins, the system retrieves from the ontology the information about the first artwork, and generates only the portion of the maze which describes this artwork and its connections with the others, namely a node containing the artwork and the pathways which represent its semantic relations with the others. Each pathway represents a relation type (e.g., story, location or agent/character), as illustrated by Figure 3 (right), where the doors leading to the pathways for "story" and "agent" are visible. When the user makes the next choice by selecting the pathway she/he wants to take, the next portion of the maze is created. If the selected pathway leads to a group of artworks (i.e., the relation it represents contains multiple artworks), the system creates an empty node whose function is to redirect the user to the single artworks, each placed in a different node (see the example in Fig. 4, right, with doors for the single

\footnotetext{
${ }^{19}$ Notice that, if the user backtracks in the same session, the nodes that have been already visited are not generated from scratch, to let the user orientate her/himself.

${ }^{20}$ The prototype ontology currently contains 1211 triples, but a wholly functioning system would be much larger.
} 


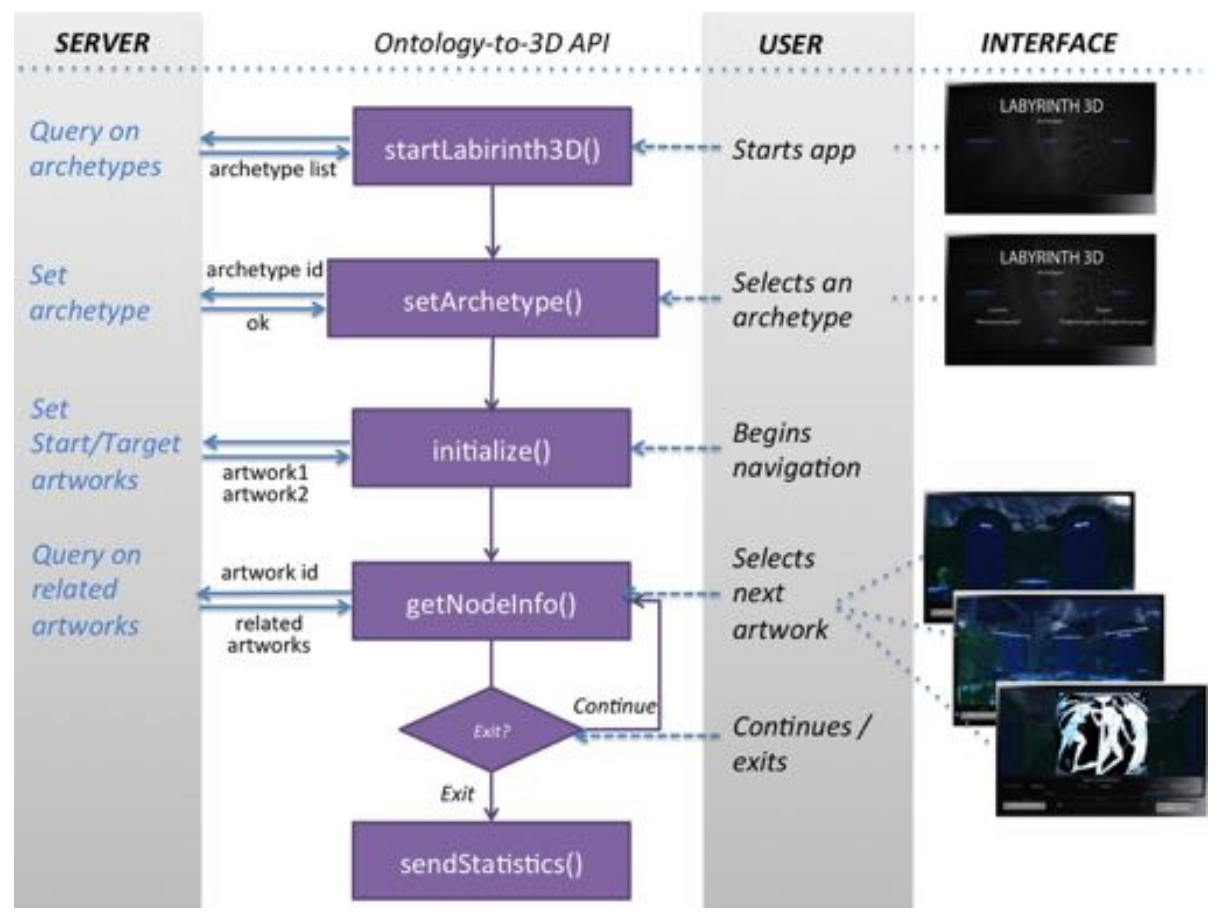

Figure 9: The interplay between the ontology server (left) and the interaction with the user (right) operated by the Ontology-to-3D API.

artworks). A direct pathway is generated only if the selected pathway leads to a single artwork. The rationale behind this strategy is to enforce the $1: 1$ mapping between artworks and nodes, so that a node always contains a single artwork.

In the following, we describe the algorithm executed by the 3D visualization module to manage the interaction with the user, supported by the ontologyto-3D API (see Figure 9). The visualization client (3D Labyrinth, bottom of figure) queries the ontology (top of the figure) through the ontology-to-3D API (see Section 5.1). The generation of the 3D labyrinth is accomplished through the following steps:

Initialization. The session begins when the user starts the application on the client device.

- Session start. First, the 3D client queries the ontology server to get the list of the available archetypes through the startLabyrinth3D() command (Fig. 2, left).

- Archetype selection. When user chooses one of the available archetypes, the client sends the selected archetype to the server (setArchetype()). 
- Navigation initialization. The client invokes an initialization command (initialize()) that selects a random pair of artworks: they provide, respectively, the initial and target nodes (Fig. 2, right). When the user clicks on the "start" command in the interface, the navigation begins.

Node generation At this point, the next node is generated, until either the user reaches the target node or she/he exits the labyrinth (by clicking the exit button posited in the navigation console). This loop is repeated each time the user selects the next artwork.

1. Retrieval of relations. The client queries the ontology to get the information about the chosen artwork (or the initial artwork at the beginning of the navigation) through the command getNodeInfo(). This command is the key to the mapping of the semantic relations encoded in the ontology onto the 3D labyrinth: given an artwork, it returns the identifier of the digital resource that represents the artwork in the media repository, its metadata (the information about its creator, title, etc.) and the list of its semantic relations (character-based, story-based, location-based relations, and so on) with the other artworks. The client employs the information about the artwork's relations with the other artworks to build the node that will contain the artwork and stores the digital resource and the information about the artwork to generate the 2D panel describing the artwork in case the user requires it (as exemplified in Fig. 3, left, Fig. 5, left, and Fig. 6).

To retrieve these data from the ontology, getNodeInfo() executes a set of SPARQL queries on the ontology, one for each possible type of semantic relations. For example, the following query extracts from the ontology the set of artworks ? $a$ that are evocative of the archetype of the labyrinth (: evokes) and display the character of Theseus (displays):

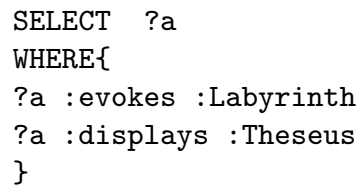

By executing similar queries for all the semantic relations embedded in the system (namely, story, character, event, location, epoch and object), the system collects all the available relations connecting the selected artwork with the rest of the repository.

2. Computation of topology. The method getNodeInfo() returns an XML fragment describing the selected artwork and its related artworks; in practice, the XML contains a section for each semantic relation type (agent, story, etc.). For example, consider the following fragment, returned by invoking the command getNodeInfo() on the artifact displayed in Fig. 5 (left), a Greek vase of the 5th century b.c. displaying Theseus killing the Minotaur: 


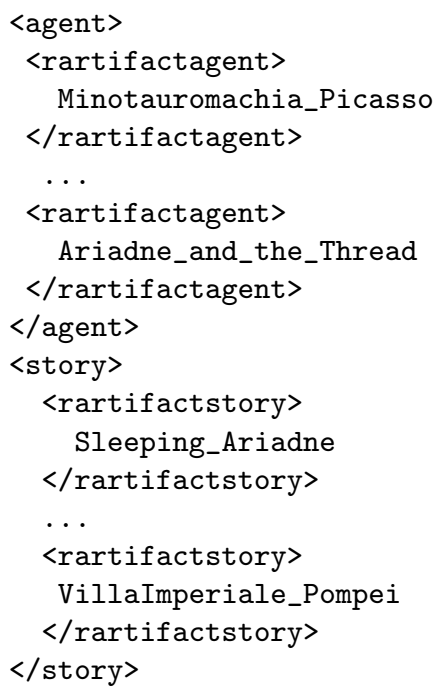

The response contains the set of artworks (or artifacts, as artworks are generically termed in $\mathrm{AO}$ ) related to the input artwork, indexed by relation types: the example shows the story relation (artworks tagged as rartifactstory) and character relation (artworks tagged as rartifactagent). The example response contains (among other artifacts not listed in the example) two story-related paintings, "Minotauromachia" by Pablo Picasso and "Ariadne and the Thread" by the Italian painter Palagio Pelagi, and two character-related artworks, namely an anonymous statue representing a sleeping Ariadne (situated at the Vatican Museums) and the frescoes depicting the myth of the Minotaur situated at the "Villa Imperiale" in Pompei. Notice that the latter two artworks are displayed in Fig 5 (right) as available alternatives after the user has chosen the story relation from the node containing the Greek vase (Fig 5, left): in the figure, they are termed, respectively "Arianna dormiente" ("Sleeping Ariadne") and "Affreschi della Villa Imperiale" ("Frescoes, Villa Imperiale").

At this point, the topology of the labyrinth is computed. For each semantic relation (in the example response: $<$ rartifactstory $>$ and $<$ rartifactagent $>$ ):

- if the relation contains a single artifact, an artwork node is created to represent it and a pathway is added from the chosen node to the new artwork node;

- if the relation contains multiple artifacts (as in the standard case), a relation node is created and a pathway is added from the chosen node to the relation node; for each artifact, then, an artwork node is created and a pathway is added from the relation node to each of the new artwork nodes (see Fig. 3, right).

3. Generation of the labyrinth. Based on the topology computed above, the next node of the $3 \mathrm{D}$ labyrinth is created and added to the $3 \mathrm{D}$ environment, 
together with its exiting pathways. When the user chooses a new artwork (either directly connected to the current one or indirectly, via a relation node), the loop is repeated.

End of session. When the user either reaches the target node or clicks on the exit button, the client executes the endLabyrinth() command to visualize the statistics of the session (time elapsed, visited nodes, etc.) and closes the session.

\section{Lesson learned}

We carried out an evaluation of the 3D interface of the system, in order to gather information about the users' liking of the system and their expectations about its use. The evaluation took place in a scientific fair, with some users taking part to participated demos and some other user freely interacting with the system. The experimentation is described in detail [41]: here, we only summarize the most important results, which are relevant for discussing the potential and the possible applications of Labyrinth 3D. 41 testers took part in the evaluation, males and females, with ages ranging from 10 to 67 years old. The system was very well welcomed by the visitors of the fair, in particular by students and teachers, who were enthusiastic about its potential for education and dissemination. The ethnographic observation of the testers who interacted directly with the system showed that the navigation was generally easy, with some problems in clicking the navigation controls when they were located far away along the pathways, because the distant controls tend to be small due to the perspective. Users were sometimes bewildered at finding themselves in a node they had already visited, but were ready to accept the explanation that this is typical of labyrinths. The users tended to read carefully the information displayed about the single items, reasoning aloud about their connection with the archetype and with the previously visited nodes. A questionnaire was given to the users to assess their liking of the system and their preferences about the use contexts. The questions about the use of the system revealed that the users would prefer the PC and the tablet for using the system, a finding that is in line with the goal of the project of creating an immersive experience. When asked about the similar media, the users selected the video game and the encyclopedia, also in line with the design goal of creating a tool for cultural dissemination. In particular, a group of 6 questions were aimed at investigating the general acceptance of the system: by using Likert scales (with 5 points from -2 to +2 , mapped onto values from $1(-2)$ to $5(+2)$ in the subsequent data analysis), we asked testers to what degree the system was: i.intuitive, ii.interesting, iii.engaging, iv.useful, v.appealing, vi. straightforward to use. The average value of the answers to the questions concerning the acceptance was 4.5, with "interesting" as the highest average value (4.7) and "straightforward" as the lowest average value (4.32), indicating that the application was appealing but that its use was not entirely clear to some users. The values are illustrated in Table 1. As it can be noticed, the standard deviation is not high, meaning that the testers generally agreed on a positive evaluation. 
Table 1: Average values for the questions about perceived properties of the system, on a 5 point Likert scale.

\begin{tabular}{|c|c|c|c|}
\hline subquestion & SYSTEM PROPERTY & AVERAGE VALUE & ST. DEV. \\
\hline i & intuitive & 4.35 & 0.72 \\
\hline ii & interesting & 4.7 & 0.57 \\
\hline iii & engaging & 4,41 & 0.74 \\
\hline iv & useful & 4.48 & 0.61 \\
\hline v & appealing & 4.5 & 0.74 \\
\hline vi & straightforward & 4.32 & 0.68 \\
\hline
\end{tabular}

The results of the evaluation suggest that the proposed approach works and open the way to a re-use of the architecture of the system for applications that leverage the creativity intrinsic to a cultural heritage archive to generate personalized paths in a 3D environment. A precondition to the reuse the approach of the Labyrinth project is to abstract the experience in the design and implementation of the system into a pipeline for creating similar applications. Given our experience in the design and implementation of Labyrinth 3D, we propose the following pipeline, divided into three phases: visual design, software development and editing, each characterized by specific professional roles. We skip the conceptual modeling phase, assuming that an annotated repository of cultural heritage objects is already available (for example, as part of some annotation project or as a by product of a digitalization initiative). It is possible, however, that, for specific projects, an ad hoc ontology is developed to satisfy this requirement: if this is the case, an ontology engineer and a domain expert cooperate to design the ontology that will constitute the backbone of the system.

The visual design phase is aimed at bridging the gap between the conceptualization of heritage objects (the archetypes in Labyrinth 3D) and the users through the use of visual and spatial metaphors (the maze in Labyrinth 3D). As argued by [13], the choice of the metaphor is crucial to communicating the conceptual model. This phase should be conducted with the help of a sample repository where a few objects have been inserted to support the design process and the subsequent development phase. Given the annotated repository, the interaction designer, in cooperation with a visual designer, i) devises a suitable metaphor for conveying the description of the objects in the repository through the 3D environment (by mapping of the object properties and their relations onto the features of the environment), ii) designs the interaction flow (specifying how the user can interact with the 3D environment and what responses he/she should get in each phase of the interaction) and iii) establishes the visual properties of the $3 \mathrm{D}$, such as its mood and appearance. A game designer may be involved in this phase to insert elements of playability into the interaction. As shown by [42], in fact, the use of game in tandem with visual metaphors increases the levels of learning. 
The software development phase translates the interaction design into 3D assets, staged and manipulated by a 3D engine. Once the interaction metaphor has been established, the 3D models that constitute the environment are created and arranged in a set of layouts by a $3 D$ production team, together with animations and camera movements (in case the navigation is achieved by constraining the user to predefined movements, as in Labyrinth 3D). In parallel, the semantic web developer implements the queries that extract the object descriptions from the ontology (previously uploaded onto an ontology server) and makes them available by programming a web service available though an API. Finally, the $3 D$ developer programs the $3 \mathrm{D}$ environment so that it implements the interaction flow established in the interaction design phase.

In the editing phase, the cultural heritage objects are collected and annotated with the semantic metadata required by the conceptual model encoded in the ontology before adding them to the repository. Metadata may include, for example, the relations of heritage objects with locations, artists, historical events, etc. Although professional annotators are preferred, metadata may be also contributed by amateurs through crowd sourcing, as recently proposed by [43].

\section{Conclusion}

In this paper, we described Labyrinth $3 \mathrm{D}$, a system where the user can explore the semantic relations over a repository of cultural objects through a virtual maze where the objects are connected by pathways representing the meaning relations over them. The approach of Labyrinth 3D leverages a systematic mapping of the conceptual model underlying the repository onto a virtual, $3 \mathrm{D}$ environment, to create an immersive and engaging experience for the user. Designed to provide an alternative to the standard approaches to archive navigation, Labyrinth 3D relies on the users' curiosity to create personal paths in a cultural domain.

In the next years, thanks to the advent of the paradigm of Linked Open Data [44], semantically encoded information about cultural heritage, including events, performances, collections, etc. will be available on the web from different sources, enabling the experimentation of new paradigms in the presentation and dissemination of cultural heritage. By applying the approach of Labyrinth 3D to the design of new applications, it will possible to refine and improve the approach described in this paper through practical case studies. The ultimate goal of this research is to take full advantage of the whole range of the new media languages, such as $3 \mathrm{D}$, to develop creative and innovative applications in the field of cultural heritage.

As a future work, we envisage the adoption of the software pipeline of Labyrinth 3D in educational projects. In this setting, in fact, our assumption is that the semantic-guided narrative exploration of themes, characters, epochs etc. can provide a ludic path to knowledge access for young students and may favour, in a gamification perspective, the process of knowledge acquisition. This would require both an extension of the current catalogue of the archetypes as 
well as an adaptation according to the specific needs of the educational project considered.

\section{Acknowledgements}

The authors wish to thank Prof. Giulio Lughi for inspiration and discussion. Our thanks go also to Neos s.r.l. for bringing to the Labyrinth project their insights and contributions.

\section{References}

[1] S. Keene, Digital collections, Routledge, 2012.

[2] A. Padilla-Meléndez, A. R. del Águila-Obra, Web and social media usage by museums: Online value creation, International Journal of Information Management 33 (5) (2013) 892-898.

[3] N. Proctor, Digital: Museum as platform, curator as champion, in the age of social media, Curator: The Museum Journal 53 (1) (2010) 35-43.

[4] W. van Hage, N. Stash, Y. Wang, L. Aroyo, Finding your way through the rijksmuseum with an adaptive mobile museum guide, The Semantic Web: Research and Applications (2010) 46-59.

[5] I. Horrocks, P. F. Patel-Schneider, F. Van Harmelen, From shiq and rdf to owl: The making of a web ontology language, Web semantics: science, services and agents on the World Wide Web 1 (1) (2003) 7-26.

[6] E. Hyvönen, Semantic portals for cultural heritage, in: Handbook on ontologies, Springer, 2009, pp. 757-778.

[7] M. Doerr, Ontologies for cultural heritage, in: Handbook on Ontologies, Springer, 2009, pp. 463-486.

[8] B. Haslhofer, A. Isaac, data. europeana. eu: The europeana linked open data pilot, in: International Conference on Dublin Core and Metadata Applications, 2011, pp. 94-104.

[9] E. Hyvönen, J. Tuominen, E. Mäkelä, J. Dutruit, K. Apajalahti, E. Heino, P. Leskinen, E. Ikkala, Second world war on the semantic web: The warsampo project and semantic portal, in: Proceedings of 14th International Semantic Web Conference, 2015.

[10] M. Doerr, S. Gradmann, S. Hennicke, A. Isaac, C. Meghini, H. van de Sompel, The europeana data model (edm), in: World Library and Information Congress: 76th IFLA general conference and assembly, 2010, pp. 10-15.

[11] M. M. Hall, From searching to using: Making sense of digital cultural heritage collections, in: Proc. of "The Search is Over!" Exploring Cultural Collections with Visualization, 2014. 
[12] M. Nitsche, Video game spaces: image, play, and structure in 3D game worlds, MIT Press, 2008.

[13] C. Ziemkiewicz, R. Kosara, The shaping of information by visual metaphors, Visualization and Computer Graphics, IEEE Transactions on 14 (6) (2008) 1269-1276.

[14] A. Warburg, Der Bilderatlas Mnemosyne, Vol. 1, Akademie Verlag, 2008.

[15] S. Thompson, Myths and folktales, The Journal of American Folklore 68 (270) (1955) 482-488.

[16] E. Hyvönen, E. Mäkelä, T. Kauppinen, O. Alm, J. Kurki, T. Ruotsalo, K. Seppälä, J. Takala, K. Puputti, H. Kuittinen, et al., Culturesampo: A national publication system of cultural heritage on the semantic web 2.0, The Semantic Web: Research and Applications (2009) 851-856.

[17] C. van den Akker, M. van Erp, L. Aroyo, R. Segers, L. Van der Meij, S. Lgene, S. G., Understanding objects in online museum collections by means of narratives, in: Proc. of the Third Workshop on Computational Models of Narrative (CMN12).

[18] W. R. Van Hage, V. Malaisé, R. Segers, L. Hollink, G. Schreiber, Design and use of the simple event model (sem), Web Semantics: Science, Services and Agents on the World Wide Web 9 (2) (2011) 128-136.

[19] N. Gershon, W. Page, What storytelling can do for information visualization, Communications of the ACM 44 (8) (2001) 31-37.

[20] J. Bruner, The narrative construction of reality, Critical inquiry (1991) $1-21$.

[21] P. Mulholland, T. Collins, Using digital narratives to support the collaborative learning and exploration of cultural heritage, in: Database and Expert Systems Applications, 2002. Proceedings. 13th International Workshop on, IEEE, 2002, pp. 527-531.

[22] P. Mulholland, T. Collins, Z. Zdrahal, Story fountain: intelligent support for story research and exploration, in: Proceedings of the 9th international conference on Intelligent user interfaces, ACM, 2004, pp. 62-69.

[23] P. Mulholland, A. Wolff, T. Collins, Curate and storyspace: an ontology and web-based environment for describing curatorial narratives, in: The Semantic Web: Research and Applications, Springer, 2012, pp. 748-762.

[24] H. Kern, J. Saward, Through the Labyrinth: designs and meanings over 5000 years, Prestel New York, 2000.

[25] P. Rosenstiehl, Labirinto, Enciclopédia Einaudi 13 (1988) 247-273. 
[26] K. Dylla, B. Frischer, P. Mueller, A. Ulmer, S. Haegler, Rome reborn 2.0: A case study of virtual city reconstruction using procedural modeling techniques, Computer Graphics World 16 (2008) 25.

[27] L. Calori, C. Camporesi, A. Negri, S. Pescarin, Virtual rome, in: SIGGRAPH Posters, ACM, 2008, p. 101.

[28] A. Guidazzoli, L. Calori, F. D. Ponti, T. Diamanti, S. Imboden, A. Mauri, A. Negri, G. B. Cohen, S. Pescarin, M. Liguori, Apa the etruscan and 2700 years of 3d bologna history, in: SIGGRAPH Asia 2011 Posters, ACM, 2011, p. 2.

[29] A. Lieto, R. Damiano, Building narrative connections among media objects in cultural heritage repositories, in: H. Koenitz, T. I. Sezen, G. Ferri, M. Haahr, D. Sezen, G. Catak (Eds.), Interactive Storytelling - 6th International Conference, ICIDS 2013, Istanbul, Turkey, November 6-9, 2013, Proceedings, Vol. 8230 of Lecture Notes in Computer Science, Springer, 2013, pp. 257-260.

[30] R. Damiano, V. Lombardo, A. Lieto, Visual metaphors for semantic cultural heritage, in: Intelligent Technologies for Interactive Entertainment (INTETAIN), 2015 7th International Conference on, IEEE, 2015, pp. 100109.

[31] R. Damiano, A. Lieto, Ontological representations of narratives: a case study on stories and actions, in: M. A. Finlayson, B. Fisseni, B. Löwe, J. C. Meister (Eds.), 2013 Workshop on Computational Models of Narrative, CMN 2013, August 4-6, 2013, Hamburg, Germany, Vol. 32 of OASICS, Schloss Dagstuhl - Leibniz-Zentrum fuer Informatik, 2013, pp. 76-93. doi:10.4230/OASIcs.CMN.2013.76.

URL http://dx.doi.org/10.4230/OASIcs.CMN.2013.76

[32] P. Lyman, B. Kahle, Archiving digital cultural artifacts, D-Lib Magazine $4(7)$.

[33] L. Aroyo, N. Stash, Y. Wang, P. Gorgels, L. Rutledge, Chip demonstrator: Semantics-driven recommendations and museum tour generation, The Semantic Web (2007) 879-886.

[34] C. Van Den Akker, S. Legêne, M. Van Erp, L. Aroyo, R. Segers, L. van Der Meij, J. Van Ossenbruggen, G. Schreiber, B. Wielinga, J. Oomen, et al., Digital hermeneutics: Agora and the online understanding of cultural heritage, in: Proceedings of the 3rd International Web Science Conference, ACM, 2011, p. 10.

[35] G. Highet, The classical tradition: Greek and Roman influences on Western literature, Oxford University Press, USA, 1949.

[36] E. T. O'Neill, Frbr: Functional requirements for bibliographic records, Library resources \& technical services 46 (4) (2002) 150-159. 
[37] D. C. M. Initiative, et al., Dublin core metadata element set, version 1.1.

[38] D. A. Norman, Affordance, conventions, and design, Interactions 6 (3) (1999) 38-43.

[39] S. Burigat, L. Chittaro, Navigation in 3d virtual environments: Effects of user experience and location-pointing navigation aids, International Journal of Human-Computer Studies 65 (11) (2007) 945-958.

[40] G. A. Miller, The magical number seven, plus or minus two: some limits on our capacity for processing information., Psychological review 63 (2) (1956) 81.

[41] R. Damiano, V. Lombardo, Labyrinth 3d. cultural archetypes for exploring media archives, Digital Creativity.

[42] L. P. Rieber, D. Noah, Games, simulations, and visual metaphors in education: antagonism between enjoyment and learning, Educational Media International 45 (2) (2008) 77-92.

[43] J. Oomen, L. Aroyo, Crowdsourcing in the cultural heritage domain: opportunities and challenges, in: Proceedings of the 5th International Conference on Communities and Technologies, ACM, 2011, pp. 138-149.

[44] C. Bizer, T. Heath, T. Berners-Lee, Linked data-the story so far, International journal on semantic web and information systems 5 (3) (2009) $1-22$. 